\title{
Vertical Use Cases in the Finnish 5G Test Network
}

\author{
Teemu Kanstrén, Jukka Mäkelä, \\ Mikko Uitto, Olli Apilo \\ VTT \\ Oulu, Finland \\ Email: firstname.lastname@vtt.fi
}

\author{
Ari Pouttu, Olli Liinamaa, Giuseppe Destino, \\ Petri Kivinen, Arto Matilainen \\ CWC, University of Oulu, \\ Oulu Finland \\ Email: firstname.lastname@oulu.fi
}

\begin{abstract}
The Finnish 5G test network (5GTN) supports development of new network technologies, and services and applications on top of these. It is developed and operated in collaboration with partners from different domains such as research, telecommunications, internet of things, software services, and data analytics. 5GTN is designed to support building products and services on top of latest and upcoming network technologies. A current example of this is $\mathbf{5 G}$ its defining properties, such as higher datarates, lower delays, and dense deployments. In this paper, we describe a set of use cases from different domain verticals, built on top of 5GTN, and the experiences so far from those use cases.
\end{abstract}

\section{INTRODUCTION}

The Finnish 5G test network (5GTN) is designed to support development and analysis of upcoming network technologies and new services on top of them. It involves partners from different domains such as network operators, network equipment, chipset designers, data analytics, and application and service developers. The 5GTN architecture is designed to support designing and executing diverse test scenarios on the network, extensive monitoring, and in-depth data analysis. Monitoring collects data from all parts of the network, including the core network, basestations, user equipment, application servers, and their integrations such as end-to-end Quality of Service $(\mathrm{QoS})$ parameters. The base 5GTN architecture is described in more detail in [5], and the monitoring and test architecture in [2]. Data analytics is provided as a service on top of all the collected data, as described in [4].

Evolution of the test network is guided by use cases and associated test scenarios from our industry partners, as well as research projects. In this paper, we describe a set of ongoing use cases on the test network, their goals, and how the test network supports them. These use cases are related to smart cars, media streaming, sports tracking, and healthcare.

The smart car use care studies 5G enabled autonomous driving and traffic support. The sports use case relates to outdoor sensors and data collection. The healthcare use case is about indoor sensoring and elderly tracking in a care home. The media use case investigates high-quality video streaming with augmented sensor information overlays. The overall 5GTN ecosystems aims to support viewpoints and needs of several partners in different domains. For example, we have prototyped healthcare services based on 5G and Internet of Things (IoT) technologies in collaboration with partners from healthcare, network and IoT domains. Such cross-domain cases are designed to give overall benefit to all parties involved, such as prototyping new services for the service providers, and realistic test environments and references for technology providers. We describe these use cases, their test architectures, and our experiences in building and running them on top of the network.

The rest of the paper is organized as follows: Section II introduces the test network and associated services. The use cases are described in Section III. Section IV discusses the results. Section V concludes this paper.

\section{Test Network Overview}

The 5GTN integrates multiple sites as shown in Fig. 1. The two main sites are located in VTT's and CWC's laboratories. Additionally, there are a number of remote sites, such as the Healthlab and car test environment shown in Fig. 1. Indoor small cell networks are deployed in these different sites. Additionally, there is outdoor macro cell coverage for the main VTT and CWC sites. These can be used for outdoor use cases, such as the sport case discussed in this paper.

The network is constantly evolving and support is added for new technologies and protocols as needed. For example, Narrowband Internet of Things (NB-IoT) and Long-Term Evolution for Machine-Type Communications (LTE-M) are two recently supported protocols for our use cases. Prototype connectivity technologies, such as proof of concept $5 \mathrm{G}$ radios, are provided to enable a realistic 5G test environment (e.g., low latency and high throughput). Beyond protocols, other technologies, such as Multi-access edge computing (MEC) [11] and evolved Multimedia Broadcast Multicast Service (eMBMS) technology [9] are also available.

MEC allows hosting third-party applications at the edges of the radio network, core network, and aggregation points. It enables low-latency applications by placing them in close proximity of the users, avoiding costly traffic round-trips. Mobile operators can also provide new services, such as location and radio information, to these MEC applications. For example, MEC enables optimization of application performance and responsiveness. eMBMS strengthens the delivery side especially on peak times, by utilizing multicast/broadcast delivery to transmit the content to multiple users simultaneously. 




Fig. 1. Network Architecture.

\section{USE CASES}

In this section we describe a representative set of ongoing use cases in 5GTN. These involve collaborations with our project partners, but also with with other projects and external partners, such as the connected car, and road weather and maintenance services with the Finnish 5G-SAFE project [8]. .

\section{A. Media Use Case}

The media use case aims to study how media applications with extremely high quality, bit rate and resolution, including video and audio, could benefit from 5G. With 5G, the content production side aims at transmitting high-speed data to uplink. The content distribution side focuses on delivering the content downlink to a massive number of clients with high quality. This use case is illustrated in Figure 2.

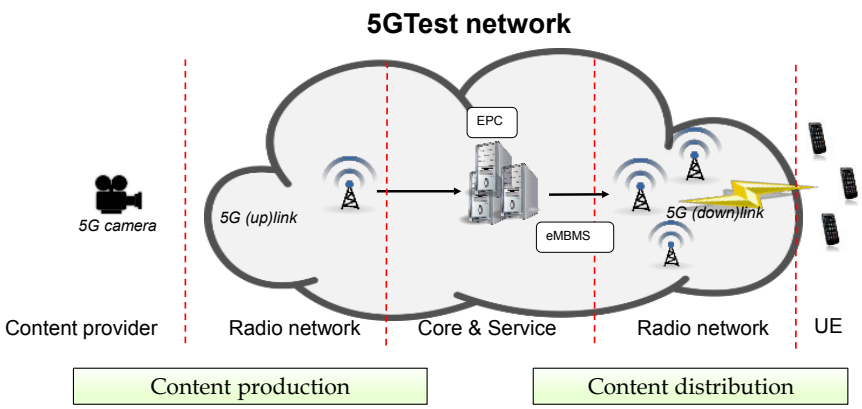

Fig. 2. $5 \mathrm{GTN}+$ Media Use Case.
One of the problems limiting content providers field use of wireless Long-Term Evolution (LTE) based cameras today is the limited uplink LTE capacity and latency. Raw or even highresolution content cannot be uploaded reliably nor real-time from the field to the transmission unit. $5 \mathrm{G}$ is expected to gain 10x uplink speed compared to the current LTE technologies, enabling high capacity. Furthermore, $5 \mathrm{G}$ is expected to reduce latency $(10 \mathrm{x})$ to $1 \mathrm{~ms}$, favoring real-time streaming. In 5GTN, we have deployed a $5 \mathrm{G}$ media content production testbed using a $5 \mathrm{G}$ proof-of-concept $(\mathrm{PoC})$ radio by Nokia for uplink, operating at $3.95 \mathrm{GHz}$ frequency, with a $200 \mathrm{MHz}$ bandwidth. The testbed holds a $4 \mathrm{~K}$ video capture camera, powerful encoding hardware, and PC clients connected to the uplink stream.

Current problems in mobile networks media distribution chain contain issues such as unequally divided output quality, limited number of simultaneous users due to unicast-style transmission, heavily loaded network cells, and poor spectrum efficiency. Each individual client requires its own unicast connection for the whole transmission path, from the core network through eNB base stations, which puts high load not only on the eNB's but also on the connection between core and access network. This makes it difficult to maintain high level QoS and Quality of Experience (QoE) for the clients.

To tackle these issues, we use eMBMS [9] in this use case. It is estimated to serve four times more users, with higher quality, compared to the general unicast approach. Traditionally, bit stream adaptation strategies, are needed in order to improve QoE against network bandwidth fluctuations [10]. These produce several representations of the input video 
with altering encoding parameters for the bit rate, allowing use of lower quality video for some users to reduce bandwidth use. eMBMS guarantees same quality for each user, and decreases the number of streams in a cell "from many to one" if only one input feed is provided. Finally, eMBMS allows spectrum to be utilized more efficiently without the necessity of using dedicated spectrum for the video.

The current goal of the media use case is to study the usefulness of eMBMS for our media producer and delivery partner in a realistic test environment. This involves components in the test network to produce high-resolution content, send it upstream to the core network through the 5G PoC radio link, and distribute this live content downlink to mobile clients (via the eMBMS). The overall performance of the network, its elements, and the end-to-end QoS and QoE of the network are monitored and analyzed. In the next phases for this use case, we will further study effects of network parameterization on the media streaming service and eMBMS.

\section{B. Connected Car}

An external car test track with LTE and ITS-5G wireless technologies located in northern part of Finland is connected to the 5GTN. Several projects use the car testing site for developing new 5G services. For example, VTT utilizes it for self driving car research in arctic conditions. Other tests include very accurate road weather forecast for autonomous cars, and pilots for enhanced logistics and road maintenance. This test environment is a joint effort between Finnish Meteorological Institute and VTT,and open for other organizations.

Connected car pilots provide a great opportunity to test delay sensitive applications using 5GTN components, such as MEC and eMBMS, in a real test environment. For example, MEC reduces the delays between the service core and incar communications, and the eMBMS has great potential for broadcasting road weather and other critical information for groups of connected vehicles.

Fig. 3 shows a typical connected car scenario where cars communicate to the network cloud via LTE and/or a $5 \mathrm{G}$ link (Uu link), and communication between cars (PC5) utilizes technologies such as 802.11p (named ITS-G5 in Europe).

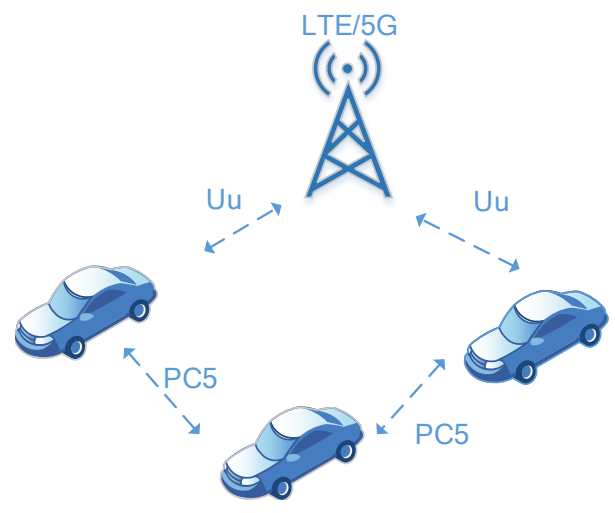

Fig. 3. Communication links in connected car scenario.

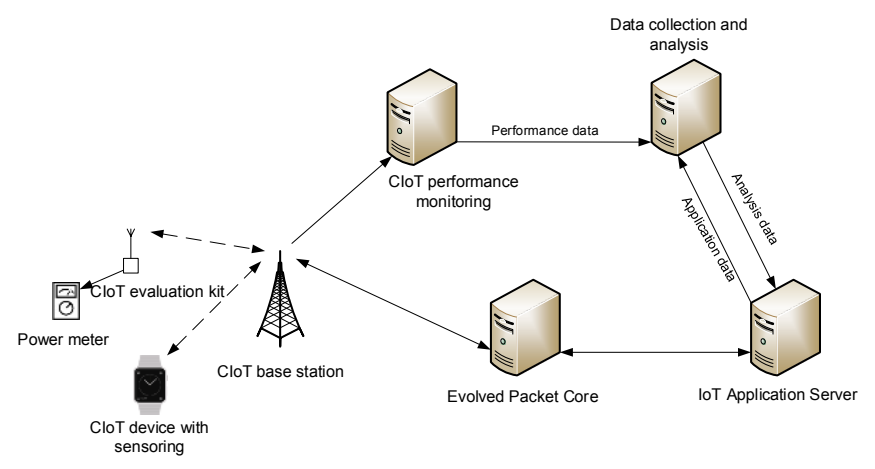

Fig. 4. 5GTN+ Sport Use Case

\section{Sport Use Case}

The 5GTN sport use case aims at evaluating the practical feasibility of cellular IoT (CIoT) connectivity for sport applications in wearable devices. This is done together with our partner with existing and upcoming products and services in the area. Typically these wearable devices measure various parameters during a physical exercise. Currently data is uploaded from a device as post-processing activity using a Universal Serial Bus (USB) cable or a low-range wireless link based on Bluetooth or Wi-Fi. CIoT enables bidirectional, longrange and real-time connectivity during exercise. This opens a completely new set of service possibilities, not only for people wearing these devices but also for other interest groups, such as coaches, personal trainers, and sports fans.

Currently the VTT 5GTN lab has both NB-IoT and LTE-M operational in two band 28 cells. Indoor cells provide a convenient environment to solve connectivity issues, and to measure the performance limits of CIoT terminals. The outdoor cell is located in northern Oulu in sub-urban and rural areas, which provides a realistic environment for field testing of sports applications.

The high-level architecture of the sport use case is shown in Fig. 4. Both CIoT evaluation kits and prototypes of CIoT wearable devices can be connected to the 5GTN using NB-IoT or LTE-M. Base station internal performance counters for the air interface are collected at the analytics server. Analytics and visualization tools are connected to the monitoring server to provide reports based on these air-interface measurements, application server measurements, and any other related data. The end-to-end traffic between the wearable application and the IoT application server runs through the 5GTN Evolved Packet Core (EPC).

Air interface measurements include throughput, hybrid automatic repeat request (HARQ) delay, and device power consumption measured at different signal levels. The signal level can be varied using tunable attenuators in the laboratory testing or moving the device within the coverage area in the outdoor testing scenario. Throughput measurements are often most convenient to be performed with CIoT evaluation kits, allowing easy generation of full buffer traffic. These kits rarely have integrated current or power monitoring and thus 
external power meters have to be used for power consumption measuring. Another interesting test scenario in this is the suitability of NB-IoT for mobile applications in terms of cell re-selection delay as it has no support for handovers [12].

CIoT improves the coverage of conventional cellular links mainly by repetitive transmissions [12]. The drawback of repetitive transmission is that it increases HARQ delays and device power consumption. One of the main targets of this use case is to measure the effect of these drawbacks in the coverage enhancement modes. The power consumption measurements close to the cell edge provide a baseline to which the simulations of the recently proposed device-todevice (D2D) relaying for IoT and wearables [13] can be compared. A realistic view of the power consumption for a given application is achieved by measuring the wearable CIoT prototypes with the power saving mode and extended discontinuous reception (DRX) enabled.

The end goal of this use case is to demonstrate a sports application running on the wearable prototype with the connectivity to the IoT application server. This should provide valuable insight on the practical end-to-end delays and other end-to-end QoS metrics, and their possible restrictions on bidirectional real-time services using the NB-IoT protocols.

\section{Health Use Case}

The health use case aims to study how IoT and 5G technologies and environments could contribute to providing better service in the home for the elderly. A special focus group are elderly people suffering from memory disorder and needing assistance for daily routines and variety of services. Service providers key challenges include, e.g., proper timing of daily care with limited resources. Currently the personnel works according to fixed schedule, which is not ideal for resource allocation nor customer experience. For instance, wake up time may vary and ideally assistance should be adapted to the morning rhythm of each individual resident. The dilemma is how working processes could be enhanced in order to enable efficient and well-timed service care on demand.

The solution explored is using IoT sensors to measure in-home consumption of electricity and water, luminance, movement, $\mathrm{CO} 2$, humidity and temperature. The sensor data is collected in the analytics databases, and analysed to gain insights on how identify different occupant characteristics, and to provide optimal services for each one.

During the service design we identified that the most valuable data is collected by water and electricity consumption sensors. Actions like the use of microwave, coffee machine, washing machine, and shower each have a unique fingerprint. The real time sensor data is monitored and analyzed by dedicated algorithms, and the results are available to care personnel. Potential extensions include additional views of results tailored for residents themselves, their relatives, the landlord and the property manager.

The high level network architecture of the health use case is illustrated in Fig. 5. The elderly home is considered as a remote site consisting of two small cell base-stations on separate floors. These pico cells are connected to the 5GTN core via Ethernet and they provide indoor coverage throughout the building. Connectivity to sensors is arranged wirelessly over NB-IoT and alternatively via local LoRa gateway.

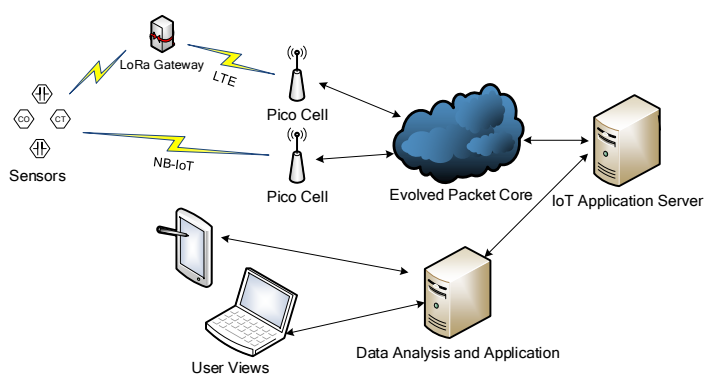

Fig. 5. 5GTN+ Health Use Case.

The target goal of this use case is to study different radio access types and protocols, sensors from several vendors, and integration of IOT platforms in a real environment and as part of a real end-user service. In the following steps for this use case, we will continue studying further how further assisted living in a smart home for the elderly can be provided.

\section{E. Inter-Continental VR Use Case}

The main objective of the inter-continental virtual reality (VR) use case is to demonstrate the potential of a 5G system to support new applications requiring high data-rate and lowlatency for a good quality of user experience. The demonstration includes an augmented reality application, 3D video recording and streaming, and 360 degree video streaming and IoT content embedded in VR, in an inter-continental setup between Finland and Korea. This demonstration is done in collaboration with the 5GCHAMPION project at the PyeongChang Winter Olympics 2018 [14], and is built on top of the 5GTN infrastructure. A high-level view of the demonstrator set-up is shown in Fig. 7.

The inter-continental sites are in Oulu, Finland, and in Gangneung, Korea. A remote VR view is provided to the Korean site from Oulu, and vice versa. The two sites are connected over a virtual private network (VPN) link. Both remote sites have two local sites as viewing places. Each viewing place has a tablet with Livesync player to control choice of viewed streams and data. The overall demonstrator set-up is shown in Fig. 6, including.

- $5 \mathrm{G} \mathrm{mmW}(27 \mathrm{GHz})$ backhaul both for last-mile broadband connectivity and moving hot-spot (bus) with beamforming

- pre-5G core networks (5GTN in Finland and proprietary ETRI core-network in Korea)

- interconnection between core-networks through VPNpipe using leased fiber optical connection

- 360 degree video equipment, IoT sensors, VR/AR user applications

The VR platform was provided by one of our Finnish partners (FINWE), enabling 360 degree video streaming in 


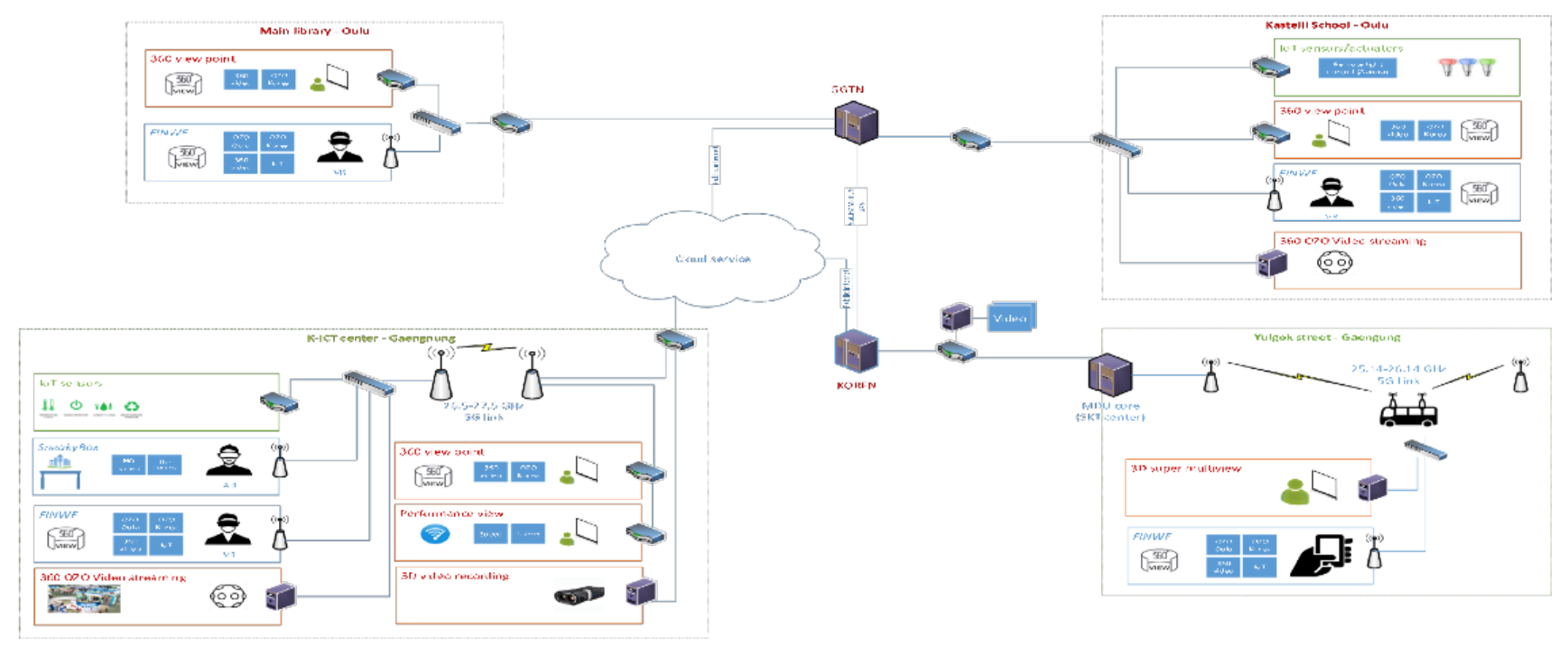

Fig. 6. Inter-continental VR use case overall setup.

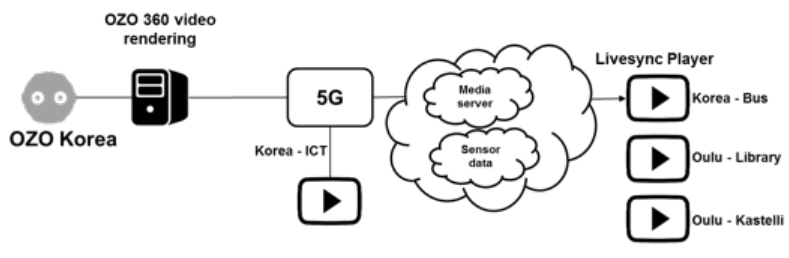

Fig. 7. $5 \mathrm{G}$ and VR components.

VR, with overlaid hotspots based on IoT data. Similarly, IoT data was provided by sensor solutions from our 5GTN IoT partners. In the Finnish side, the Kastelli school and Oulu city library provide citizens access to local VR viewing sites, and Kastelli school also with its own VR OZO camera, and IoT hotspot data.

The Korean site consisted of the K-ICT Center and Yulkok Street in Gangneung, Korea. This site deployed 5G radio technology to demonstrate $5 \mathrm{G}$ backhauling performance with $27 \mathrm{GHz}$ hybrid beamforming using 64 antenna elements. The K-ICT site focused on demonstrating AR and VR applications, along with integrated IoT sensors and actuators. The Yulgok street site focused on demonstrating a moving $5 \mathrm{G}$ hotspot.

The goal of this use case was to provide means for our partners to showcase potential $5 \mathrm{G}$ applications alongside a major global event, and across different continents. This includes the technologies such as high-definition VR streams combined with real-life IoT data, remote interaction, and high-speed remote streaming. Interested parties include network operators, IoT device/service providers, and telecommunication infrastructure vendors. That is, besides technology development and testing support, the test networks can also provide useful demonstration platforms.

\section{DISCUSSION}

To support detailed analytics, various monitoring and analytics tools have been integrated into the network. These are described in more detail in our previous works ([1], [2], [4]). For monitoring this includes tools such as InfluxDB timeseries database for data storage, Grafana for visualization, Qosium for end-to-end QoS measurements (packet loss, jitter, delay, throughput), EXFO EPC monitoring (core EPC signaling), test control flow data (e.g., data amount sent/received by test clients), node resources (e.g, application server load), and application specific measurements (e.g., connected clients). Different tools are used depending on the use case requirements, and new ones added as needed.

For data analysis, we start with basic visualizations such as illustrated in Fig. 8. For deeper analytics, we use statistical and machine learning algorithms over all the combined data to find properties of interest, as discussed in [3].



Fig. 8. Example results.

As an example, Fig. 8 illustrates a few measurements for a test scenario at the VTT lab site, demonstrating aspects of both the media and sport use cases. In the media use case part, two high throughput video streams were streamed over the 5G PoC link in the uplink direction to two clients, establishing two unicast video sessions. UHD (4K) camera captured H.264 encoded and MPEG-TS encapsulated UDP 
streams, with bitrates of $90 \mathrm{Mbps}$ and $30 \mathrm{Mbps}$, were utilized for streams 1 and 2, respectively. In Fig. 8 the video client and server nodes in the network (top graph) illustrate the throughputs for the server (green) and client 1 (yellow). The wireless end-to-end delay for server and client 1 is illustrated in the second graph from top.

The second part of the scenarios illustrated in Fig. 8 consisted of sensors reporting temperature readings using NB-IoT, while tracking User Equiment (UE) network idle periods at the EPC. The figure shows the temperature measurements (bottom graph) and the core network active/inactive UE status (second graph from bottom). This type of information is useful for studying properties such as power consumption, network activity, and different power saving modes of the devices.

Beyond such visualizations, we can apply more in-depth analysis such as machine learning methods as we described in [3]. To enable this, we treat each test run as a labeled test set. Varying the test parameters and labels allows producing large data sets well suited for such analytics.

While seemingly simple, defining the test scenarios and goals for new and upcoming technologies itself has proven to be one of the biggest challenges. Understanding what effects new emerging technologies will have on a business requires foresight, deep understanding of the domain and of what the technologies enable. This is complex and requires time and iterations. Thus these first test scenarios have mostly focused on the enabling factors of the new network technologies. For example, the media use case on improved throughput for high number of clients, with high quality video streams. Similarly, the sport case on prototyping use of the new IoT communications technologies from the UE to the server.

As an example of more advanced analytics involving multiple variables and their interactions, we have studied a network use case in 5GTN, investigating the impact of network configurations on spectral efficiency. These tests focused on network architecture and power level changes, varying these across test executions, and collecting extensive monitoring data. This could then be fed to visualizations and machine learning algorithms to better understand impacts and optimization opportunities between the different variables, and how indoor network spectral efficiency cloud be optimized.

\section{CONCLUSIONS}

5GTN is an ongoing project with several partners in Finland. The use cases we have described here represent some of the main use cases currently being deployed on the test network, in collaboration with our project partners. In addition, we provide access to the test network and related expertise to interested third parties. In the practice of building, operating, and offering the test network as a service we have observed a very high complexity in providing a highly useful and reliable test network, as well as the in-depth expertise to perform, design, and analyze the use cases on top of it. Yet such networking technologies and the ability to understand them indepth form a core component as infrastructure for building the next generations of intelligent systems, making them crucial for developing new products and services. As such, we expect these types of test environments to become even more relevant in the future, and keep evolving and improving our test network and the services provided on top of it.

\section{ACKNOWLEDGMENT}

This paper is carried out in the $5 \mathrm{GTN}+$ project, partially funded by Tekes, the Finnish funding agency for innovation. The authors would like to thank the whole consortium for all the help and cooperation. Parts of the inter-continental VR case have also been performed as part of the EU H2020 5GCHAMPION [15] project.

\section{REFERENCES}

[1] T. Kanstrén and J. Perälä, "Testing Technologies in Finnish 5G Test Network", ETSI User Conf. on Advanced Automated Testing (UCAAT), 2015.

[2] T. Kanstrén et al., "Testing Technologies to Support Network and Services Testing in a 5G Test Network", 12th Adv. Int'l. Conf. on Telecom. (AICT), Valencia, Spain, 2016.

[3] T. Kanstrén, J. Liikka, J. Mäkela, M. Luoto, J. Prokkola, "Preliminary Big Data in a 5G Test Network", IEEE Workshop on Big Data and Machine Learning in Telecom, Washington DC, USA, 5-8 december 2016.

[4] T. Kanstrén, "Experiences in Testing and Analysing Data Intensive Systems", IEEE International Conference on Software Quality, Reliability and Security (QRS, industry track), 2017.

[5] E. Piri et al., "5GTN: A Test Network for 5G Application Development and Testing", European Conf. on Networks and Communications (EuCNC), Athens, Greece, 2016.

[6] http://5gtn.fi/, "5GTN - 5G Test Network", Oulu, Finland, [retrieved: January, 2018].

[7] http://5gtnf.fi/, "5G Test Network Finland", Finland, [retrieved: January, 2018].

[8] http://5gsafe.fmi.fi, "5G-SAFE road safety enhancement enabled by 5G", Finland, [retrieved: January, 2018].

[9] 3GPP, "Enhanced Television Services Over 3GPP eMBMS", 2017, Available at http://www.3gpp.org/images/PDF/EnTV_article_for_3GPP_Web_ site_v8.pdf.

[10] O. Oyman and S. Singh, "Quality of Experience for HTTP Adaptive Streaming Services", IEEE Communications Magazine, IEEE, 50(4):2027, 2012.

[11] ETSI, Mobile Edge Computing (MEC); Framework and Reference Architecture, ETSI standard GS MEC 003 V1.1.1, 2016.

[12] O. Liberg, M. Sundberg, Y.-P. E. Wang, J. Bergman, and J. Sachs, Cellular Internet of Things: Technologies, Standards, and Performance. London, UK: Academic Press, 2018.

[13] M. Höyhtyä, O. Apilo, and M. Lasanen, "Review of latest advances in 3GPP standardization: D2D communication in 5G systems and its energy consumption models," Future Internet, vol. 10, no. 1, Jan. 2018.

[14] M. Mueck, et al., "5G CHAMPION - Rolling out 5G at 2018 Winter Olympic Games", IEEE Global Communications Conference (GlobeCom), 2016 .

[15] http://www.5g-champion.eu/, "5G Communication with a Heterogeneous, Agile Mobile network in the Pyeongchang wInter Olympic competitioN", [retrieved: February, 2018]. 